\title{
ISOLATED SINGULARITIES OF QUADRATIC DIFFERENTIALS ARISING FROM A MODULE PROBLEM
}

\author{
JEFFREY CLAYTON WIENER
}

\begin{abstract}
If $R \subset S$ are Riemann surfaces, we will say that $z_{0} \in S-R$ is an isolated point boundary component of $R$ if there exists a neighborhood $U$ of $z_{0}$ in $S$ such that $U-\left\{z_{0}\right\} \subset R$. We prove that the quadratic differential $Q(z) d z^{2}$ obtained by solving the module problem $P\left(a_{1}, \ldots, a_{k}\right)$ applied to a free family of homotopy classes on $R$ can be extended to $z_{0} \in S$ so that either $Q(z)$ is regular at $z_{0}$ or $Q(z)$ has a simple pole at $z_{0}$.
\end{abstract}

Introduction. Let $R$ denote an open Riemann surface. A free family of homotopy classes on $R$ is a set of nontrivial homotopy classes $H_{i}, i$ $=1, \ldots, k$, such that all classes $H_{i}$ are distinct, no class $H_{i}$ is the class of a point cycle [3, p. 40], and there exist $k$ Jordan curves $h_{i} \in H_{i}, i=1, \ldots, k$, no two of which have a common point. Take $k$ nonnegative values $a_{1}, \ldots, a_{k}$ so that $a_{1}+\cdots+a_{k} \neq 0$. Let $P\left(a_{1}, \ldots, a_{k}\right)$ denote the module problem associated with a free family of $k$ homotopy classes on $R$ (see Definition 2). J. A. Jenkins and N. Suita [4] proved that for the module problem $P\left(a_{1}, \ldots, a_{k}\right)$ there exists a quadratic differential $Q(z) d z^{2}$ on $R$ with the property that the trajectories of $Q(z) d z^{2}$ (see [4, Theorem 1] or Theorem 1 for a precise statement of this result) divide $R$ into a family of doublyconnected domains $D_{i}, i=1, \ldots, k$, such that

$$
\iint_{R}|Q(u)| d A_{u}=\sum_{i=1}^{k} a_{i}^{2} M_{i},
$$

where $M_{i}$ is the module of $D_{i}$.

Let $S \subset R$ denote a Riemann surface. Suppose that $z_{0}$ is a point of $S-R$ such that there exists a neighborhood $U$ of $z_{0}$ on $S$ with $U-\left\{z_{0}\right\} \subset R$. We will show that $Q(z) d z^{2}$ can be extended to $z_{0} \in S$ so that either $Q(z)$ is regular at $z_{0}$ or $Q(z)$ has a simple pole at $z_{0}$.

1. Prelimaries. The following three definitions appear in [4, pp. 106-110].

Definition 1. By a free family of homotopy classes $H$ on a Riemann surface $R$ we mean a set of homotopy classes $H_{i}, i=1, \ldots, k$, such that:

(1) all classes $H_{i}$ are distinct and nontrivial;

Received by the editors February 6, 1975.

AMS (MOS) subject classifications (1970). Primary 30A52; Secondary 30A15, 30A38.

Key words and phrases. Homotopy class, point cycle, module, module problem, quadiatic differential, extremal metric, canonical exhaustion, Huber module. 
(2) there exist Jordan curves $h_{i} \in H_{i}, i=1, \ldots, k$, no two of which have a common point;

(3) no class $H_{i}$ is the class of a point cycle [3, p. 40].

Let $R$ denote a Riemann surface and $H$ a free family of $k$ homotopy classes $H_{1}, \ldots, H_{k}$ on $R$. Let $a_{1}, \ldots, a_{k}$ denote $k$ nonnegative numbers, not all zero. We now define what we mean by the module problem $P\left(a_{1}, \ldots, a_{k}\right)$ on $R$ associated with $H$.

Definition 2. Let $\rho(u)|d u|$ denote a conformally invariant metric (linear density) on $R$ such that, for any rectifiable arc $c$ in the coordinate neighborhood of a local (uniformizing) parameter, $\int_{c} \rho(u)|d u|$ exists. Suppose that $\iint_{R} \rho^{2} d A_{u}$ exists and is finite. If for each $i=1, \ldots, k$ and every locally rectifiable $h(i) \in H_{i}, \int_{h(i)} \rho(u)|d u| \geqslant a_{i}$, then the metric $\rho(u)|d u|$ will be called admissible for the module problem $P\left(a_{1}, \ldots, a_{k}\right)$. The extremal problem consists of finding the greatest lower bound $M\left(a_{1}, \ldots, a_{k}\right)$ of $\iint_{R} \rho^{2} d A_{u}$ as $\rho(u)|d u|$ ranges over all admissible metrics. If the greatest lower bound is obtained for a particular metric the latter is called an extremal metric for the problem.

An extremal metric is uniquely determined up to sets of measure zero. For this reason, if an extremal metric exists, it is called the extremal metric for the module problem.

Definition 3. By an admissible family of doubly-connected domains associated with a free family of homotopy classes $H_{i}, i=1, \ldots, k$, on a Riemann surface $R$, we mean a finite set of domains $D_{i(j)}, j=1, \ldots, l$, $l \leqslant k, i(j)<i\left(j^{\prime}\right)$ for $j<j^{\prime}, 1 \leqslant i(j) \leqslant k$, on $R$ such that:

(1) no two domains $D_{i(j)}, j=1, \ldots, l$, have a common point;

(2) a simple closed curve in $D_{i(j)}$ separating the boundary components of $D_{i(j)}$ belongs to $H_{i(j)}$ when given the appropriate sense.

For a class $H_{i}$ to which no doubly-connected domain is assigned we may say that the corresponding domain $D_{i}$ is degenerate and we assign to $D_{i}$ the module zero. With this interpretation we may assume that the index in Definition 3 is always $i=1, \ldots, k$.

A bordered Riemann surface $\bar{R}$ is a one dimensional complex manifold with boundary $\partial \bar{R}$. A finite Riemann surface is a compact bordered Riemann surface.

The definition of a free family of homotopy classes on a finite bordered Riemann surface $\bar{R}$ [2, pp. 440-441] allows homotopy classes of arcs with end points on $\partial \bar{R}$. In the course of such a homotopy the end points of the arc in question are permitted to move on their respective (not necessarily distinct) boundary components.

Definition 4. By a free family of homotopy classes on a finite bordered Riemann surface we mean a set of homotopy classes $H_{i}, i=1, \ldots, k$, where $H_{i}, i=1, \ldots, j$, say, are homotopy classes of simple closed curves and $H_{i}$, $i=j+1, \ldots, k$, are homotopy classes of arcs joining boundary components (either set may be empty) such that:

(1) all classes $H_{i}, i=1, \ldots, k$, are distinct and nontrivial;

(2) no class $H_{i}, i=1, \ldots, k$, consists of closed curves homotopic to a point of $\bar{R}$;

(3) there exist simple closed curves $h_{i} \in H_{i}, i=1, \ldots, j$, and $\operatorname{arcs} h_{i} \in H_{i}$, 
$i=j+1, \ldots, k$, on $\bar{R}$ no two of which have a common point.

We shall say that a quadrangle $D$ on $\bar{R}$ is associated with the homotopy class $H_{i}, i=j+1, \ldots, k$, if a pair of opposite sides of $D$ lie, respectively, on $\partial \bar{R}$ joined by arcs in $H_{i}$ and if the class of arcs lying in $D$ and joining these sides is contained in $H_{i}$.

By an admissible family of domains $D$ associated with a free family of homotopy classes $H_{i}, i=1, \ldots, k$, on $R$ we mean a family of domains (quadrangles and doubly-connected domains) each associated with a class $H_{i}$ and not more than one associated with any such class. Furthermore, we require that no two domains in the family have a point in common. Finally, for a class $H_{i}, i=1, \ldots, k$, to which no domain has been associated in the family $D$, we say that the corresponding domain is degenerate and has module zero.

The definitions of $P\left(a_{1}, \ldots, a_{k}\right)$ and $M\left(a_{1}, \ldots, a_{k}\right)$ are the same as above.

2. Known results. The following theorem was proven by Jenkins and Suita [4, Theorem 1].

THEOREM 1. Let $R$ denote a Riemann surface and $H_{i}, i=1, \ldots, k$, a free family of homotopy classes on $R$. Then for the module problem $P\left(a_{1}, \ldots, a_{k}\right)$ there exists an extremal metric of the form $|Q(u)|^{1 / 2}|d u|$ where $Q(u) d u^{2}$ is a regular quadratic differential on $R$.

For an admissible family of domains $D_{i}, i=1, \ldots, k$, associated with the free family of homotopy classes,

$$
\sum_{i=1}^{k} a_{i}^{2} M_{i} \leqslant M\left(a_{1}, \ldots, a_{k}\right),
$$

where $M_{i}$ is the module of $D_{i}$.

The next theorem considers a free family of homotopy classes on a finite Riemann surface [2, Theorem 1].

THEOREM 2. Let $\bar{R}$ denote a finite bordered Riemann surface on which are given a finite number (possibly zero) of distinguished points. Let $R^{\prime}$ be obtained from $\bar{R}$ by deleting these distinguished points. Let $H_{i}, i=1, \ldots, k$, be a free family of homotopy classes on $R^{\prime}$. Then for the module problem $P\left(a_{1}, \ldots, a_{k}\right)$ there exists an extremal metric $|Q(u)|^{1 / 2}|d u|$ where $Q(u) d u^{2}$ is a quadratic differential on $\bar{R}$ regular apart from possible simple poles at the distinguished points.

Provided that neither is $\bar{R}$ a closed Riemann surface of genus 1 nor is $\bar{R}-\partial \bar{R}$ a doubly-connected domain (in either case without any distinguished points), the trajectories of $Q(u) d u^{2}$ which have limiting end points at its finite critical points, together with those which pass through distinguished points, divide $\bar{R}$ into an admissible family $D$ of domains $D_{i}, i=1, \ldots, k$, associated with the given free family of homotopy classes $H_{i}$. If $M_{i}$ is the module of $D_{i}$, then

$$
\iint_{\bar{R}}|Q(z)| d A_{z}=M\left(a_{1}, \ldots, a_{k}\right)=\sum_{i=1}^{k} a_{i}^{2} M_{i} .
$$


3. Result. We now define an isolated point boundary component of an open Riemann surface.

Definition. Let $R$ be an open Riemann surface. Suppose that there is a Riemann surface $S \subset R$ and a point $z_{0} \in S-R$ such that there exists a neighborhood $U$ of $z_{0}$ in $S$ so that $U-\left\{z_{0}\right\} \subset R$. Then $z_{0}$ will be called an isolated point boundary of $R$.

Let $R$ denote an open Riemann surface and suppose that $z_{0}$ is an isolated point boundary component of $R$. Using Theorem 1 we conclude that there exists an extremal metric on $R$ of the form $|Q(u)|^{1 / 2}|d u|$, where $Q(u) d u^{2}$ is a regular quadratic differential on $R$, associated with the module problem $P\left(a_{1}, \ldots, a_{k}\right)$ for a free family of homotopy classes $H_{i}, i=1, \ldots, k$, on $R$.

For suitable definition of local uniformizing parameters at $z_{0}, S=R \cup$ $\left\{z_{0}\right\}$ is a Riemann surface. We will prove that $Q(u)$ can be extended to $z_{0} \in S$ so that either $Q(u)$ is regular at $z_{0}$ on $S$ or $Q(u)$ has a simple pole at $z_{0}$ on $S$.

Let $\left\{R_{n}\right\}$ denote a canonical exhaustion of $S$ with $z_{0} \in R_{n}-\partial R_{n}$ for each $n$. We may assume that $H_{i}, i=1, \ldots, k$, determines a free family of homotopy classes $H_{i n}, i=1, \ldots, k$, on each $R_{n}-\left(\left\{z_{0}\right\} \cup \partial R_{n}\right)$. Using Theorem 2, we conclude that there exists a quadratic differential $Q_{n}(z) d z^{2}$ with at worst a simple pole at $z_{0}$ such that $\left|Q_{n}(z)\right|^{1 / 2}|d z|$ provides the extremal metric for the module problem $P\left(a_{1}, \ldots, a_{k}\right)$ on $R_{n}$.

Let the corresponding decomposition of $R_{n}$ into an admissible family of domains associated with $H_{i n}, i=1, \ldots, k$, be given by $D_{i n}, i=1, \ldots, k$, and let $M_{i n}$ denote the module of $D_{i n}$. It is known that for fixed $i, M_{i n}$ is uniformly bounded by $\pi$ times the reciprocal of the Huber module on $R$ for the class $H_{i}$ (see [3, pp. 42-43]), the latter quantity being finite since $H_{i}$ is nontrivial and not the class of a point cycle.

Let $D_{i}, i=1, \ldots, k$, denote any admissible family of domains on $R$ associated with $H_{i}, i=1, \ldots, k$, and let $M_{i}$ denote the module of $D_{i}$. Using Theorem 1,

$$
\sum_{i=1}^{k} a_{i}^{2} M_{i} \leqslant M\left(a_{1}, \ldots, a_{k}\right)<\infty .
$$

Since $H_{i n}, i=1, \ldots, k$, is a free family of homotopy classes determined by $H_{i}$ on $R_{n}-\left(\partial R_{n} \cup\left\{z_{0}\right\}\right)$ and $R_{n+1} \supset R_{n}$, we can think of each curve in $H_{i n}$ as a curve in $H_{i n+1}$, which in turn is a curve in $H_{i}$. With this interpretation $\left(H_{i n} \subset H_{i n+1}\right.$ and $H_{i n} \uparrow H_{i}$ for fixed $\left.i\right)$ it should be clear that $M_{i n} \rightarrow M_{i}$ as $n \rightarrow \infty$. Using Theorem 2 ,

$$
\iint_{R_{n}}\left|Q_{n}(z)\right| d A_{z}=\sum_{i=1}^{k} a_{i}^{2} M_{i n} \rightarrow \sum_{i=1}^{k} a_{i}^{2} M_{i}<\infty .
$$

So $\left\{\iint_{R_{n}}\left|Q_{n}(z)\right| d A_{z}\right\}$ is uniformly bounded.

Let $(U, \phi)$ denote a parametric disc on $S$ containing $z_{0}$, that is, $U$ is an open subset of $S$ containing $z_{0}$ and $\phi$ is a homeomorphism of $U$ onto $\{|z|<1\}$ such that $\phi\left(z_{0}\right)=0$. In $U, Q_{n}(z)=h_{n}(z)+\beta(n) / z$, where $h_{n}(z)$ is regular in $U$ and $\beta(n)$ is a complex number. In fact, we can assume that $h_{n}(z)$ is regular on the closure of $U$, denoted by $\mathrm{Cl} U$.

We will show that a subsequence of $\{\beta(n)\}$ converges. Note that 


$$
\beta(n)=\frac{1}{2 \pi i} \int_{|z|=r} Q_{n}(z) d z, \quad r<1 .
$$

So $|\beta(n)| \leqslant(1 / 2 \pi) \int_{0}^{2 \pi}\left|Q_{n}\left(r e^{i \theta}\right)\right| r d \theta$. Integrating from $r=0$ to $r=1$,

$$
\begin{aligned}
|\beta(n)| & \leqslant \frac{1}{2 \pi} \iint_{\mathrm{Cl} U}\left|Q_{n}\left(r e^{i \theta}\right)\right| r d r d \theta \\
& =\frac{1}{2 \pi} \iint_{\mathrm{Cl} U}\left|Q_{n}(z)\right| d A_{z} \leqslant M\left(a_{1}, \ldots, a_{k}\right) / 2 \pi<\infty .
\end{aligned}
$$

So there exists a subsequence of $\{\beta(n)\}$ which converges to $\beta,|\beta|<\infty$. We continue to designate this subsequence by $\{\beta(n)\}$. Now

$$
\begin{aligned}
\int_{\mathrm{Cl} U}\left|h_{n}(z)\right| d A_{z} & =\iint_{\mathrm{Cl} U}\left|Q_{n}(z)-\beta(n) / z\right| d A_{z} \\
& \leqslant \int_{\mathrm{Cl} U}\left|Q_{n}(z)\right| d A_{z}+\int_{0}^{2 \pi} \int_{0}^{1}|\beta(n)| d r d \theta \\
& =\int_{\mathrm{Cl} U}\left|Q_{n}(z)\right| d A_{z}+2 \pi|\beta(n)|,
\end{aligned}
$$

which is uniformly bounded on $\mathrm{Cl} U$. As was done in [4, Lemma 1], we can conclude that $\left|Q_{n}(z)-\beta(n) / z\right|$ is uniformly bounded on compact subsets of $U$.

By Weierstrass's theorem, there exists a subsequence $\left\{Q_{n(s)}(z)\right\}$ of $\left\{Q_{n}(z)\right\}$ such that $Q_{n(s)}(z)-\beta(n(s)) / z$ converges to a regular function $f(z)$ on $U$. So $Q_{n(s)}(z) \rightarrow f(z)+\beta / z=Q(z)$ on $U$. Thus, either $Q(z)$ is regular in $U$ $(\beta=0)$ or $Q(z)$ is regular in $U-\left\{z_{0}\right\}$ and has a simple pole at $z_{0} \in S$.

\section{BIBLIOGRAPHY}

1. J. A. Jenkins, Univalent functions and conformal mapping, 2nd ed., Springer-Verlag, Berlin and New York, 1965.

2. _ On the existence of certain general extremal metrics, Ann. of Math. (2) 66(1957), 440-453. MR19, 845.

3. J. A. Jenkins and N. Suita, On analytic self-mappings of Riemann surfaces, Math. Ann. 202(1973), 37-56.

4. __ On analytic self-mappings of Riemann surfaces. II, Math. Ann. 209(1974), 109-115.

Department of Mathematics, Georgia Institute of Technology, Atlanta, Georgia 30332 